\title{
ALG3-CDG: lethal phenotype and novel variants in Chinese siblings
}

\author{
Yue Bian ${ }^{1,2}$. Chong Qiao, ${ }^{1,2}$ ShuGuang Zheng ${ }^{3}$ Hao Qiu ${ }^{4} \cdot$ Huan $\mathrm{Li}^{1} \cdot$ ZhiTao Zhang $^{1}$ - ShaoWei Yin ${ }^{1}$. \\ HongKun Jiang ${ }^{5} \cdot$ Jesse Li-Ling ${ }^{6}$ CaiXia Liu ${ }^{1,2} \cdot$ Yuan Lyu $\mathbb{C}^{1,2}$
}

Received: 13 February 2020 / Revised: 17 June 2020 / Accepted: 28 June 2020 / Published online: 12 July 2020

(c) The Author(s), under exclusive licence to The Japan Society of Human Genetics 2020

\begin{abstract}
Congenital disorders of glycosylation (CDG) are a group of genetic, mostly multisystem disorders, which often involve the central nervous system. ALG3-CDG is one the some 130 known CDG. Here we report two siblings with a severe phenotype and intrauterine death. Whole-exome sequencing revealed two novel variants in ALG3: NM_005787.6:c.512G>T (p. Arg171Leu) inherited from the mother and NM_005787.6:c.511C >T (p.Arg171Trp) inherited from the father.
\end{abstract}

\section{Introduction}

Congenital disorders of glycosylation (CDG) are a group of inherited disorders characterized by deficient glycosylation [1]. They are mostly multisystem disorders often involving the central nervous system [2-5]. ALG3-CDG is a rare disorder, with only 23 patients reported so far [2-13]. ALG3 adds the 6th mannose to the growing dolichol-linked oligosaccharide in the ER $[2,14]$. Here we report two siblings with a severe malformation syndrome and intrauterine death.

Yuan Lyu

hawk.1v@163.com

1 Department of Obstetrics and Gynecology, Shengjing Hospital of China Medical University, Shenyang, China

2 Key Laboratory of Maternal-Fetal Medicine of Liaoning Province, Key Laboratory of Obstetrics and Gynecology of Higher Education of Liaoning Province, Research Center of China Medical University Birth Cohort, Shenyang, China

3 Department of Radiology, The People's Hospital of China Medical University, Shenyang, China

4 Dian Diagnostics Group Co., LTD., Hangzhou, China

5 Department of Pediatrics, The First Affiliated Hospital of China Medical University, Shenyang, China

6 Jinxin Research Institute of Reproductive Medicine and Genetics, Jinjiang Maternal and Children's Health Care Hospital, Chengdu, China

\section{Materials and methods}

\section{Genetic analysis}

Genomic DNA was extracted from the muscle tissue of the fetuses and from peripheral blood of their parents. Wholeexome sequencing (WES) and Sanger sequencing were performed according to standard procedures.

\section{Western blotting}

Muscle tissues from the patients and a control were washed with phosphate-buffered saline and western blot analysis was performed according to standard procedures.

\section{Results}

\section{Clinical report}

We report two siblings with severe multisystem dysmorphism who were born to healthy parents. The mother stated that she was never exposed to alcohol, warfarin, or other drugs. The present study was approved by the hospital's Institutional Review Board, and written informed consent was obtained from the parents. The control tissue was obtained from a healthy fetus who was born with induced labor. The tissue sample was voluntarily donated to our hospital, which is in accordance with the ethical guidelines.

\section{Patient 1}

Patient 1 was a male fetus. Multiple congenital abnormalities were detected on prenatal ultrasound scan (USS) at 26 weeks 
A

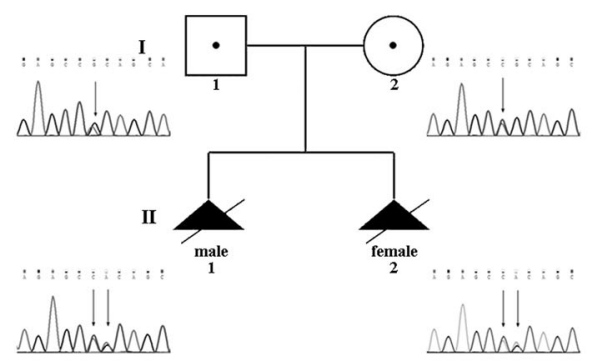

B

C
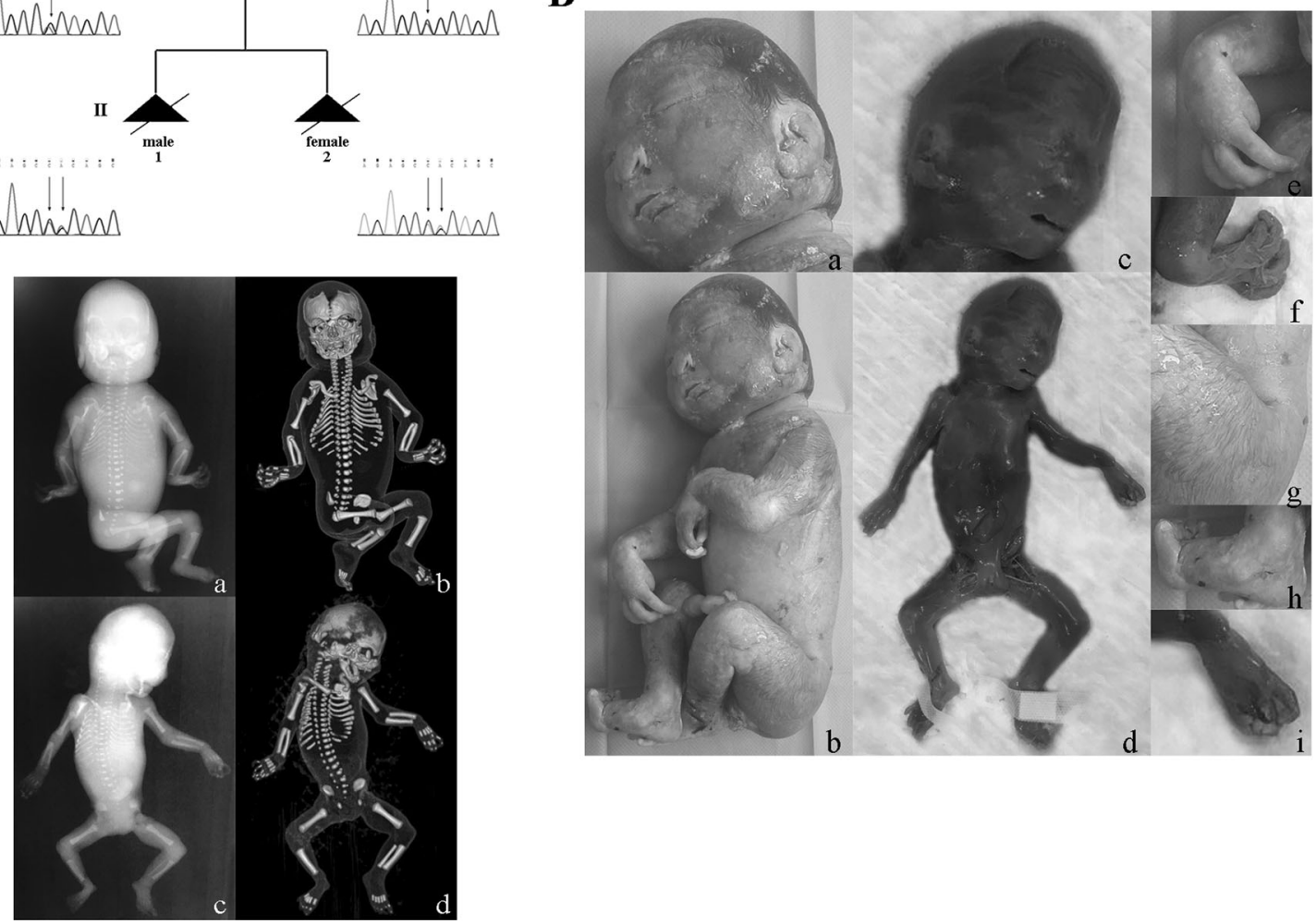

Fig. 1 Pedigree, photographs, and radiographic examination results of the two patients. a II 1: patient 1 (the first pregnancy of the family); II 2: patient 2 (the second pregnancy of the family). b a, b, e, g, h

Pictures of patient 1. c, d, f, i Pictures of patient 2. c a, b Radiographic examination result of patient 1 . c, d Radiographic examination result of patient 2

of gestation. The parents decided to terminate the pregnancy at 28 weeks of gestation. The following multiple anomalies were detected: intrauterine growth restriction with short limbs, micrognathia, separation of bilateral renal collecting system, hydramnios, the hands postural fixation, increased cardiothoracic proportion, and fetal edema especially of the scalp tissue. Facial dysmorphism included eyelid ptosis, hypertelorism, short nose, flat nasal bridge, anteverted nares, thin lips, low-set, large and thick ears with malformed pinnae and micrognathia (Fig. 1b (a)). In addition, the patient had a short neck, short limbs, inverted widely spaced nipples; joint contractures, long fingers and toes, ulnar deviation of fingers, adducted thumbs, toes valgus, abnormal subcutaneous fat distribution at neck and buttock, and hirsutism (Fig. 1b (b, $\mathrm{e}-\mathrm{h})$ ). Radiographic examination showed short long bones with wide metaphyses, short vertebral bodies, short ribs, a narrow thorax, paddle-shaped iliac wings, and bilateral small scapulae (Fig. 1c (a, b)). Fetal karyotype was normal.

\section{Patient 2}

This patient was the sister of patient 1 and the mother's second pregnancy. USS at 18 weeks of gestation showed

scalp tissue edema and mildly short limbs. After genetic counseling, the parents decided to terminate the pregnancy at 22 weeks of gestation. Dysmorphism was similar to that in patient 1. Contractures of wrists, knees, and left elbow were milder. Hirsutism and short neck were not observed (Fig. 1b (c, d)). Radiographic examination revealed short limbs, short ribs, a narrow thorax, vertebral hypoplasia, and scoliosis (Fig. 1c (c, d)).

\section{Whole-exome sequencing and Sanger sequencing results}

Whole-exome sequencing showed two novel variants in the ALG3 gene: NM_005787.6:c.512G>T (p.Arg171Leu) inherited from the mother and NM_005787.6:c.511C $>$ T (p. Arg171Trp) inherited from the father. The second patient showed the same variants. (Fig. 1a). The effect of NM_005787.6:c.512G>T (p.Arg171Leu) was predicted using the computational tools Sorting Intolerant from Tolerant (SIFT), PolyPhen-2, and MutationTaster, M-CAP, and the results indicated that the protein variant is "damaging," "damaging," "disease causing," and "damaging," respectively. The CADD_phred score of the variant is 35. SIFT, 
A
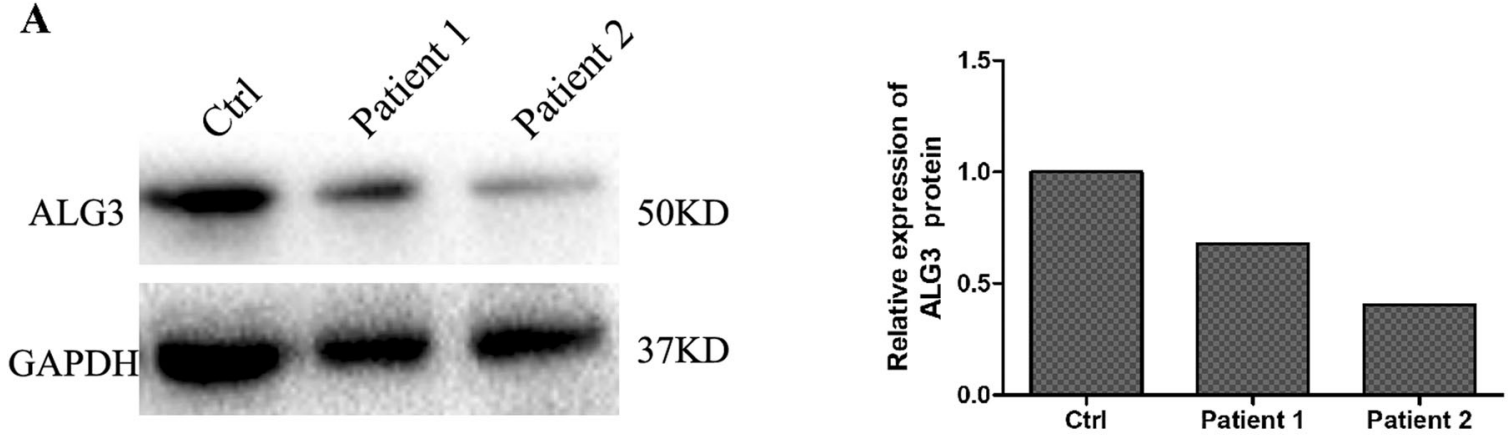

B

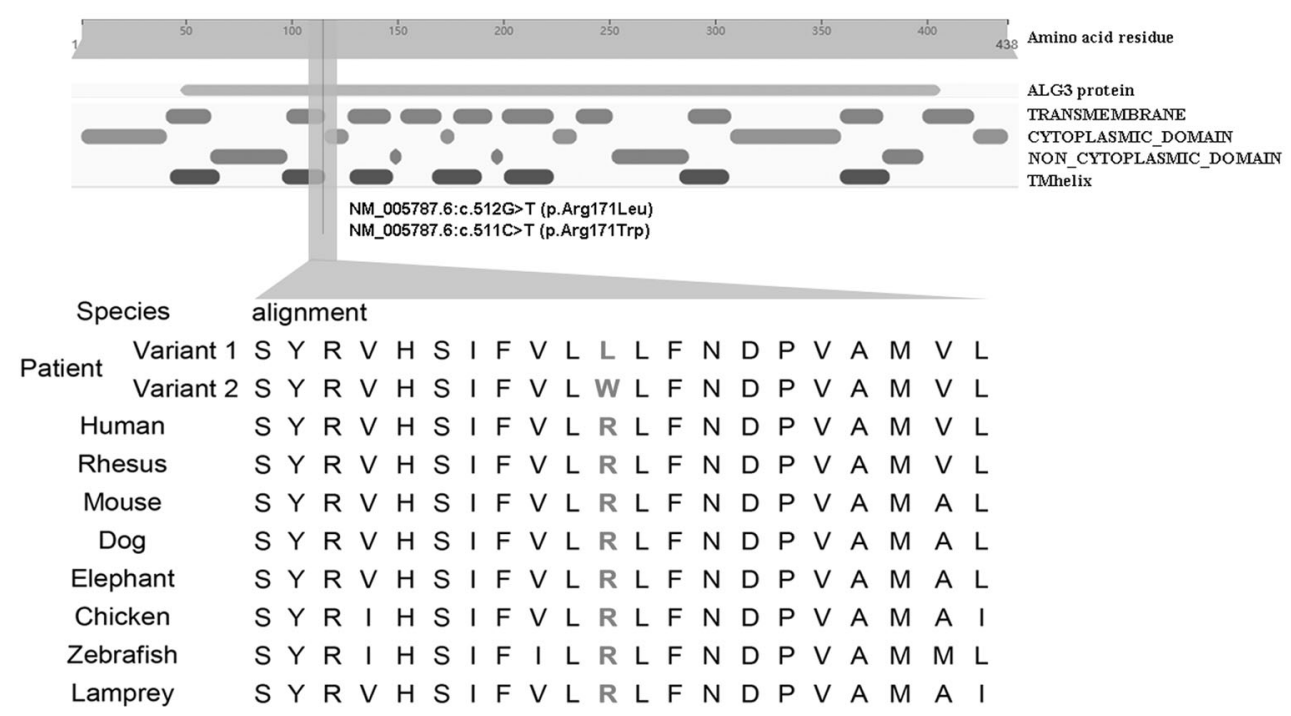

Fig. 2 The effects of the variants on ALG3 protein. a Relative protein expression of ALG3 in the two patients. GAPDH was used as an internal reference protein. b The location of the variants and conservation of ALG3 residues. The variants were located in ALG3 protein domain, and the amino acid residue was highly conserved
PolyPhen-2, MutationTaster and M-CAP predicted NM_005787.6:c.511C>T (p.Arg171Trp) to be a "damaging," "damaging," "disease causing," and "damaging" variant, respectively. The CADD_phred score of this variant is 33. According to the ACMG criteria, both variants were classified as uncertain significance (PM2, PM5 and PP3).

\section{ALG3 protein expression in the two patients}

Western blot analysis of ALG3 in muscle tissues showed a clearly decreased expression in the two patients compared to that in the control (Fig. 2a).

\section{Discussion}

Twenty-three ALG3-CDG patients have been reported so far. These patients showed a broad phenotypical spectrum that mainly includes dysmorphism and neurological, cardiac, skeletal, gastrointestinal, and urogenital abnormalities (summary of reported patients in Table 1). The phenotypes of our patients were already extremely severe in utero and resembled those of the patients reported by Lepais et al. [3] and Sun et al. [10]. The patient described by Sun et al. died at 19 days and showed multiple dysmorphisms with prominent cerebral, cerebellar, skeletal, cardiac, pulmonary, urogenital, hepatic, and pancreatic involvement. Lepais et al. [3] reported similarly affected siblings. In one, the abnormalities were detected at 23 weeks of gestation, which was terminated at 25 weeks. In the other, intrauterine growth retardation and short long bones were noted at 13 weeks. The parents refused to terminate the pregnancy, and the child died at 12 days due to heart failure. This severe fetal phenotype resembles that of a patient reported with ALG9-CDG (OMIM: 608776) and patients with Schneckenbecken dysplasia, also known as SLC35D1-CDG (OMIM: 269250).

In conclusion, our cases expand the gene variant database of ALG3-CDG. In addition, we provide a new prenatal genotype-phenotype correlation of ALG3-CDG, which may be beneficial for the future prenatal diagnosis of other CDG cases. 


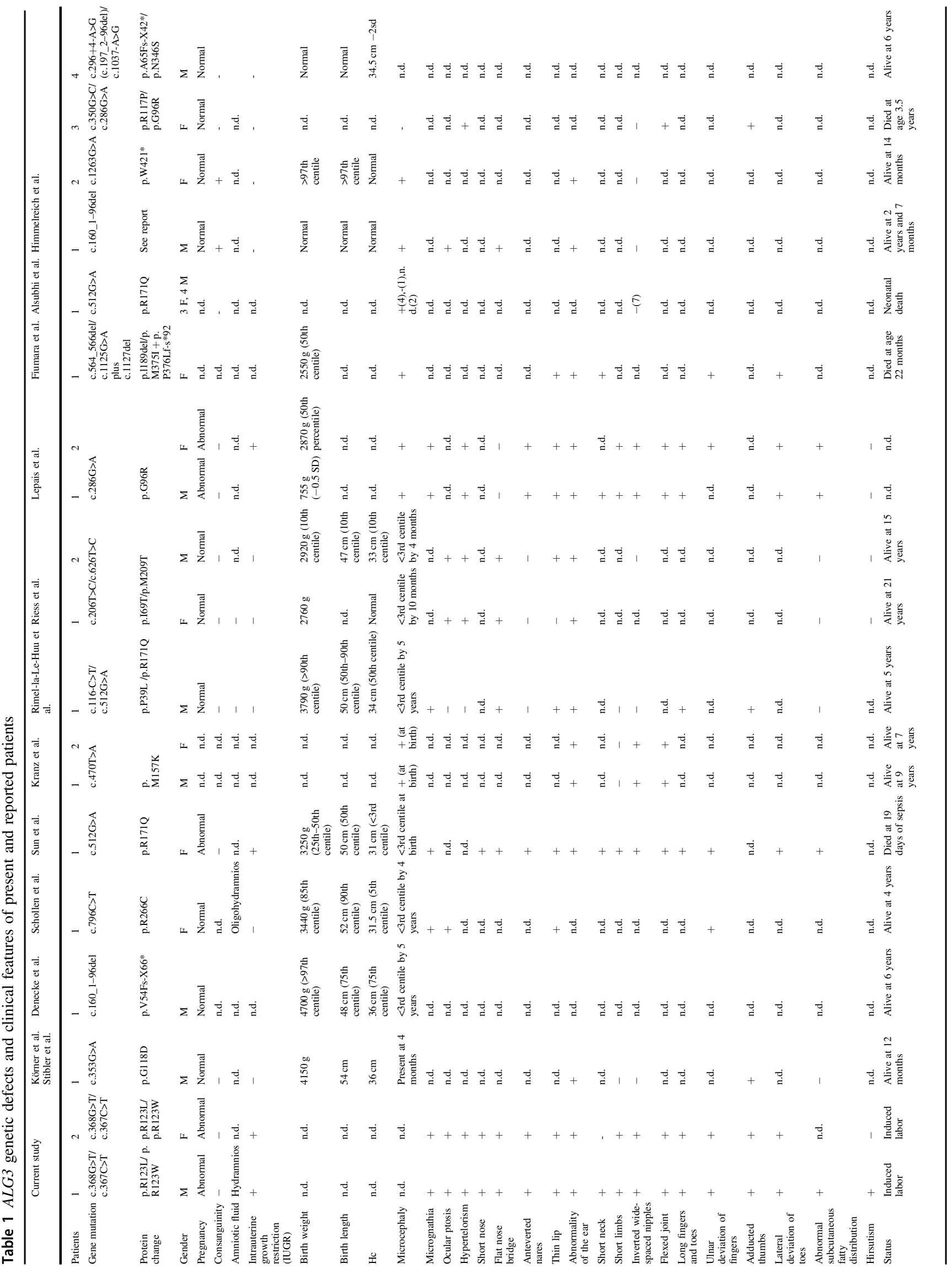


Acknowledgements We are grateful to the patients and their family for providing samples and clinical histories.

Funding This research was supported in part by the 345 Talent Project to YL and National Natural Science Foundation of China (81701462 to YL) and National Key R\&D Program of China (2018YFC1002900 to YL).

Author contributions YB and SGZ performed the research and analyzed and interpreted the data. YB and HQ drafted the manuscript. HL, ZTZ, and YSW helped in sample collection. CXL performed phenotyping of patients. YL and CQ helped in analysis and interpretation of ES data. HKJ provided technical support. YB, HL, ZTZ, YSW, YL, JLL, and CQ were involved in scientific discussion and offered suggestions. CQ and YL conceived and designed the study, revised the manuscript, and provided final approval of the manuscript.

\section{Compliance with ethical standards}

Conflict of interest The authors declare that they have no conflict of interest.

Publisher's note Springer Nature remains neutral with regard to jurisdictional claims in published maps and institutional affiliations.

\section{References}

1. Chang IJ, He M, Lam CT. Congenital disorders of glycosylation. Ann Transl Med. 2018;6:477.

2. Rimella-Le-Huu A, Henry H, Kern I, Hanquinet S, Roulet-Perez E, Newman CJ. et al.Congenital disorder of glycosylation type Id (CDG Id): phenotypic, biochemical and molecular characterization of a new patient. J Inherit Metab Dis. 2008;31:S381-S6.

3. Lepais L, Cheillan D, Frachon SC, Hays S, Matthijs G, Panagiotakaki E, et al. ALG3-CDG: report of two siblings with antenatal features carrying homozygous p.Gly96Arg mutation. Am J Med Genet A. 2015;167A:2748-54.

4. Kranz C, Sun L, Eklund EA, Krasnewich D, Casey JR, Freeze HH. CDG-Id in two siblings with partially different phenotypes. Am J Med Genet A. 2007;143A:1414-20.

5. Alsubhi S, Alhashem A, Faqeih E, Alfadhel M, Alfaifi A, Altuwaijri W, et al. Congenital disorders of glycosylation: the Saudi experience. Am J Med Genet A. 2017;173:2614-21.

6. Denecke J, Kranz C, von Kleist-Retzow JC, Bosse K, Herkenrath $\mathrm{P}$, Debus $\mathrm{O}$, et al. Congenital disorder of glycosylation type Id: clinical phenotype, molecular analysis, prenatal diagnosis, and glycosylation of fetal proteins. Pediatr Res. 2005;58:248-53.

7. Körner C, Knauer R, Stephani U, Marquardt T, Lehle L, von Figura K. Carbohydrate deficient glycoprotein syndrome type IV: deficiency of dolichyl-P-Man:Man(5)GlcNAc(2)-PP-dolichyl mannosyltransferase. EMBO J. 1999;18:6816-22.

8. Riess S, Reddihough DS, Howell KB, Dagia C, Jaeken J, Matthijs G, et al. ALG3-CDG (CDG-Id): clinical, biochemical and molecular findings in two siblings. Mol Genet Metab. 2013;110:170-5.

9. Stibler H, Stephani U, Kutsch U. Carbohydrate-deficient glycoprotein syndrome-a fourth subtype. Neuropediatrics. 1995;26:235-7.

10. Sun L, Eklund EA, Chung WK, Wang C, Cohen J, Freeze HH. Congenital disorder of glycosylation id presenting with hyperinsulinemic hypoglycemia and islet cell hyperplasia. J Clin Endocrinol Metab. 2005;90:4371-5. 
11. Fiumara A, Barone R, Del Campo G, Striano P, Jaeken J. Electroclinical features of early-onset epileptic encephalopathies in congenital disorders of glycosylation (CDGs). JIMD Rep. 2016;27:93-9.

12. Himmelreich N, Dimitrov B, Geiger V, Zielonka M, Hutter A-M, Beedgen $\mathrm{L}$, et al. Novel variants and clinical symptoms in four new ALG3-CDG patients, review of the literature, and identification of AAGRP-ALG3 as a novel ALG3 variant with alanine and glycine-rich N-terminus. Hum Mutat. 2019;40:938-51.
13. Schollen E, Grünewald S, Keldermans L, Albrecht B, Körner C, Matthijs G. CDG-Id caused by homozygosity for an ALG3 mutation due to segmental maternal isodisomy UPD3(q21.3-qter). Eur J Med Genet. 2005;48:153-8.

14. Denecke J, Kranz C, Kemming D, Koch H-G, Marquardt T. An activated 5' cryptic splice site in the human ALG3 gene generates a premature termination codon insensitive to nonsense-mediated mRNA decay in a new case of congenital disorder of glycosylation type Id (CDG-Id). Hum Mutat. 2004;23:477-86. 